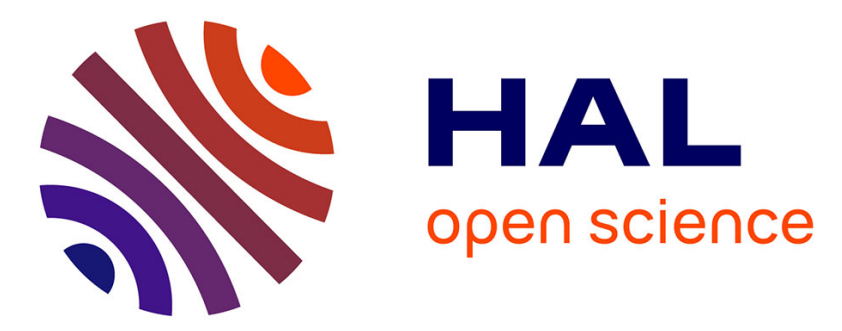

\title{
Utilisation du pessaire gynécologique en cas de prolapsus génital: une enquête auprès des internes
}

Mathilde Martin-Lasnel, Krystel Nyangoh-Timoh, Raffaèle Fauvet, Anne-Cécile Pizzoferrato

\section{- To cite this version:}

Mathilde Martin-Lasnel, Krystel Nyangoh-Timoh, Raffaèle Fauvet, Anne-Cécile Pizzoferrato. Utilisation du pessaire gynécologique en cas de prolapsus génital: une enquête auprès des internes. Gynécologie Obstétrique Fertilité \& Sénologie, 2021, 49 (10), pp.763-766. 10.1016/j.gofs.2021.03.030 . hal-03191958

\section{HAL Id: hal-03191958 \\ https://hal.science/hal-03191958}

Submitted on 21 Apr 2021

HAL is a multi-disciplinary open access archive for the deposit and dissemination of scientific research documents, whether they are published or not. The documents may come from teaching and research institutions in France or abroad, or from public or private research centers.
L'archive ouverte pluridisciplinaire HAL, est destinée au dépôt et à la diffusion de documents scientifiques de niveau recherche, publiés ou non, émanant des établissements d'enseignement et de recherche français ou étrangers, des laboratoires publics ou privés. 
Utilisation du pessaire gynécologique en cas de prolapsus génital : une enquête auprès des internes

The use of pessaries for treatment of pelvic organ prolapse : a survey from gynecologists and urologists residents

Utilisation du pessaire en cas de prolapsus

Mathilde Martin-Lasnel ${ }^{\mathrm{a}}$, Krystel Nyangoh-Timoh ${ }^{\mathrm{b}}$, Raffaèle Fauvet ${ }^{\mathrm{a}, \mathrm{c}}$, Anne-Cécile

Pizzoferrato $^{\mathrm{a}}$

aDépartement de Gynécologie-Obstétrique, Hôpital Universitaire de Caen Normandie, Caen,

France

${ }^{\mathrm{b}}$ Departement of Obstetrics and Gynecology, Rennes Hospital. Rennes, University 1, Rennes,

France ; INSERM, LTSI - UMR 1099

cUniversité Caen Normandie, Inserm U1086 «ANTICIPE », Unité de Recherche

Interdisciplinaire pour la Prévention et le Traitement des Cancers, Axe 2 : Biologie et

Thérapies Innovantes des Cancers Localement Agressifs (BioTICLA)

Auteur correspondant :

Mathilde Martin Lasnel

Département de Gynécologie-Obstétrique,

Hôpital Universitaire de Caen Normandie

Avenue Côte de Nacre, 14033 Caen Cedex, France

E-mail : martin.mathilde2@gmail.com

\section{Résumé :}


INTRODUCTION : Le prolapsus des organes pelviens (POP) est une affection fréquente, responsable de symptômes altérant significativement la qualité de vie des femmes. Malgré son efficacité, son faible coût et ses effets indésirables mineurs, le pessaire est peu utilisé en France. L'objectif de notre étude était d'évaluer les connaissances, la formation et les pratiques des internes concernant l'utilisation du pessaire.

MATERIEL ET METHODES : Cette enquête par questionnaire a été mené auprès des internes en gynécologie obstétrique, gynécologie médicale et urologie français entre mars et septembre 2020 .

RESULTATS : Au cours de la période d'étude, 328 internes ont répondu au questionnaire. La majorité d'entre-eux $(52,1 \%)$ a rapporté ne jamais avoir assisté à une consultation spécialisée en pelvi-périnéologie ; seulement 31,7 \% se sentaient à l'aise avec la mise en place d'un pessaire. Selon eux le pessaire était indiqué dans 3 principales situations : en cas de contreindication à la chirurgie $(80 \%)$, dans l'attente d'une chirurgie $(79 \%)$ et chez les femmes âgées de plus de 70 ans (62\%). Le pessaire pouvait être proposé à toutes les femmes pour seulement 46,9\% d'entre eux. Près de 53\% des internes rapportaient ignorer les principales complications des pessaires et $83,5 \%$ estimaient avoir besoin d'une formation complémentaire sur le sujet.

CONCLUSION : Les internes semblent globalement sensibilisés à l'utilisation du pessaire. Leur connaissance concernant les indications, les complications ou encore la surveillance nous laisse penser qu'il est essentiel de promouvoir leur formation afin que le pessaire fasse partie intégrante de l'arsenal thérapeutique de première intention en cas de POP. INTRODUCTION: Pelvic organ prolapse (POP) is a common condition r 


\section{Abstract ;}

INTRODUCTION: Pelvic organ prolapse (POP) is a common condition responsible for symptoms that significantly affect the quality of life in women. Despite its effectiveness, low cost and minor side effects, the pessary is little used in France. The objective of our study was to assess the knowledge, training and practices of residents regarding pessaries.

MATERIAL AND METHODS : This survey was conducted among residents in obstetrics gynecology, medical gynecology and urology in France between March and September 2020.

RESULTS: During the study period, 328 interns responded to the questionnaires. The majority of residents $(52.1 \%)$ reported never having attended a consultation specializing in pelvicperineology. Only $31.7 \%$ felt comfortable having a pessary inserted. According to them, the pessary was indicated in 3 main situations: in case of contraindication to surgery $(80 \%)$, while awaiting surgery $(79 \%)$ and in women over 70 years old $(62 \%)$. The pessary could be offered to all women for only $46.9 \%$ of them. Almost $53 \%$ of residents reported ignoring the main complications of pessaries and $83.5 \%$ felt they needed further training on the subject.

CONCLUSION: Interns seem to be generally aware of the use of the pessary. Their knowledge of the indications, complications or even monitoring leads us to believe that it is essential to promote their training so that the pessary becomes an integral part of the first-line therapeutic arsenal in the event of POPs.

Mots Clés : pessaire, prolapsus des organes pelviens, médecin en formation Keys words : pessaries, pelvic organ prolapse, residents 


\section{INTRODUCTION}

Le prolapsus des organes pelviens (POP) de la femme est une affection fréquente avec une prévalence variant entre 31,8 et $97,7 \%$ d'après l'examen clinique respectant la classification Pelvic Organ Prolapse Quantification (1). Il peut être responsable de symptômes altérant significativement la qualité de vie des femmes $(2,3)$. Seuls les POP symptomatiques doivent faire l'objet d'une prise en charge thérapeutique (4). Bien que la promontofixation coelioscopique soit considérée comme la technique chirurgicale de référence en cas de prolapus apical, de récentes études démontrent une morbidité non négligeable de cette chirurgie $(5,6)$. La voie vaginale donne des résultats fonctionnels comparables à la promotofixation mais avec plus de risque de complication en cas de voie vaginale avec prothèse ou plus de récidive en cas de voie vaginale autologue. Malgré son efficacité, son faible coût et ses effets indésirables mineurs, l'utilisation du pessaire reste peu répandue $(7,8)$. Or il constitue une véritable alternative entre expectative et chirurgie. Différentes études exposent les pratiques des professionnels utilisant le pessaire aux Etats-Unis, au Royaume Unis, aux Pays-Bas ou encore au Brésil (9-14), et une seule récente en France (15). Dans l'attente d'étude randomisée sur le sujet, il parait nécessaire d'évaluer les pratiques des professionnels impliqués dans la prise en charge des patientes présentant un POP notamment celle des médecins en formation. L'objectif de notre étude était d'évaluer les connaissances, la formation et les pratiques des internes concernant les pessaires.

\section{MATERIEL ET METHODE}

Nous avons réalisé cette enquête auprès des internes en gynécologie obstétrique, gynécologie médicale et urologie français. Pour se faire, nous avons créé un questionnaire via la plateforme LIMESURVEY. Nous avons ensuite contacté l'ensemble des représentants de chaque spécialité de chaque ville française afin qu'ils diffusent notre questionnaire à 
l'ensemble des internes concernés entre mars 2020 et septembre 2020. Le questionnaire se divisaient en sept catégories : les données démographiques des internes interrogés, leur pratiques et leurs connaissances en matière d'indication ou de non-indication d'utilisation du pessaire, de choix du type de pessaire, d'éducation, de suivi, d'échec et de complications mais également leur éventuel besoin en formation (Annexe 1).

L'analyse statistique a été descriptive en donnant pour chaque variable les effectifs et la proportion de chaque modalité dans l'échantillon.

\section{RESULTATS}

Sur la période de l'étude, 328 internes ont répondu aux questionnaires avec un taux de réponse complète à $87,2 \%$ (286/328). Cent seize internes étaient en première partie d'internat $(38,9 \%)$; et 182 en deuxième partie $(61,1 \%)$.

La majorité des internes $(52,1 \%)$ a rapporté ne jamais avoir assisté à une consultation spécialisée en pelvi-périnéologie (171/328). Parmi eux, 49,4\% ont déclaré avoir déjà mis en place des pessaires (162/328). Seulement $31,7 \%$ se sentaient à l'aise avec la mise en place d'un pessaire. Ils proposaient le pessaire en première intention pour 53,3 \%. La mise en place d'un pessaire était indiquée selon eux dans 3 principales situations : en cas de contreindication à la chirurgie (80\%), dans l'attente d'une chirurgie (79\%) et chez les femmes âgées de plus de 70 ans (62\%). Seulement 61 internes le proposaient aux femmes de moins de 50 ans (18\%). Pour 154 internes, le pessaire pouvait être proposé à toutes les femmes (46,9\%). L'antécédent d'hystérectomie n'était pas une contre-indication à la pose du pessaire pour $79,5 \%$ d'entre eux. La majorité $(67,4 \%)$ considérait que le pessaire pouvait être proposé aux patientes ayant une activité sexuelle. Concernant l'utilisation d'un traitement oestrogénique locale chez les patientes ménopausées, $41 \%$ des internes n’y étaient pas favorables ou n'avaient pas avis. Le pessaire donut, le pessaire anneau et le pessaire cube étaient les trois 
principaux pessaires utilisés par les internes (respectivement $49,4 \%, 47,2 \%$ et $45,1 \%$ ). Pour $82,6 \%$ des internes, l'éducation des patientes à la pose et au retrait du pessaire devrait être réalisée en consultation. Concernant le rythme de surveillance du pessaire, 51,5\% des internes déclaraient qu'il fallait l'adapter en fonction du mode d'utilisation de la patiente (nettoyage ou non par la patiente, utilisation permanente ou intermittente) mais 33,5\% estimaient qu'une surveillance tous les 3 à 6 mois serait plus justifiée. La béance vulvaire $(53,9 \%)$, l'incontinence urinaire démasquée $(47,2 \%)$, l'obésité $(49,1 \%)$ et le prolapsus extériorisé $(39,9 \%)$ constitueraient les principaux facteurs de risque d'échec du pessaire. Près de $53 \%$ des internes rapportaient ignorer les principales complications des pessaires. En ce qui concerne leur formation, 41,8\% n'avait jamais entendu parler du pessaire au cours de leur cursus et 83,5\% (274/328) estimaient avoir besoin d'une formation complémentaire sur le sujet.

\section{DISCUSSION}

A l'heure où il existe une forte remise en question des techniques chirurgicales utilisant du matériel prothétique, la prise en charge conservatrice par pessaire connaît un regain d'intérêt en France parmi les professionnels. Il nous a donc paru licite d'évaluer les pratiques et les connaissances des internes en ce qui concerne l'utilisation du pessaire chez les femmes présentant un POP. En effet le pessaire, pourtant utilisé depuis le XVIIIe, semble redevenir une solution thérapeutique de choix. En France actuellement, ses indications ne sont pas clairement définies et son utilisation reste souvent réservée aux femmes âgées, en cas de contre-indication ou de refus de chirurgie (7). Dans notre enquête, les internes interrogés privilégiaient effectivement l'utilisation du pessaire dans ses indications et seulement $18 \%$ d'entre eux le proposaient aux femmes de moins de 50 ans. Or il permet une amélioration significatives des symptômes, avec des taux de satisfaction variant entre 58 et $90 \%$ selon les études (16-18) et un taux complications peu élevé et en général peu sévères (19). 
Dans d'autres pays, l'utilisation du pessaire est plus large. Aux Etats-Unis, Cundiff et al. ont publié une étude en 2000 réalisée auprès des membres de l'American Urogynecologic Society. Parmi l'ensemble des praticiens interrogés, $77 \%$ utilisaient le pessaire comme traitement de première intention (9). Chez les femmes présentant un POP symptomatique, toutes les alternatives possibles doivent être expliquées, l'intérêt majeur du pessaire étant sa réversibilité contrairement à la chirurgie.

Concernant l'utilisation du pessaire en cas d'antécédent d'hystérectomie, $80 \%$ des internes interrogés considéraient que cela n'était pas une contre-indication à l'utilisation du pessaire. Sur ce point dans la littérature, les avis divergent. Cundiff et al. retrouvaient des résultats similaires puisque moins de la moitié des répondants considérait qu'un antécédent d'hystérectomie était une contre-indication à l'utilisation d'un pessaire. En revanche, Yimphona et al. en 2018 (20), Markle et al. en 2011(21) ou encore Mutone et al. en 2005 (22) retrouvaient que l'antécédent d'hystérectomie était associé à l'échec.

L'activité sexuelle des patientes semblent ne pas constituer une contre-indication pour la majorité des internes interrogés dans notre étude. Ces résultats sont cohérents à ceux retrouvés dans la littérature. En effet de nombreuses études ont démontré qu'il n'y avait pas plus d'échec ou d'abandon chez les femmes sexuellement actives $(23,24)$. Voir, pour Brincat et al., les femmes sexuellement actives étaient plus susceptibles d'utiliser le pessaire de façon prolongée $(\beta=2,204 ; p=0,021)$ et cela quelle que soit l'indication de la pose du pessaire (25). Certains auteurs, comme Fernando et al. ont montré une amélioration significative de la sexualité avec l'utilisation du pessaire. En effet, 17\% de leurs patientes avaient signalé une augmentation de la fréquence de l'activité sexuelle $(\mathrm{p}=0,001)$ et $11 \%$ rapportaient une amélioration de leur satisfaction sexuelle $(\mathrm{p}=0,04)(26)$. Une autre étude, publiée par Kuhn et al. en 2009, rapportait également une amélioration significative du désir ( $p<0,01)$, de la 
lubrification $(p<0,001)$ et de la satisfaction sexuelle $(p<0,001)$ après mise en place d'un pessaire chez des femmes ayant un POP symptomatique (27).

Les pessaires donut et anneaux sont les deux types les plus utilisés par les internes interrogés. En effet le pessaire anneau semble être le plus souvent proposé du fait de sa facilité d'insertion (12). Cundiff et al. rapportaient que $78 \%$ des praticiens interrogés utilisaient le pessaire anneau en cas de prolapsus de l'étage moyen ou antérieur et plutôt le pessaire Donut en cas de prolapsus de l'étage postérieur. En effet, l'adaptation du type de pessaire en fonction du type de prolapsus est une véritable question ; ce d'autant que la taille optimale n'est pas toujours déterminée à la première pose et que le pessaire n'est pas remboursé à l'heure actuelle en France. Dans une étude prospective, Ding et al. ont étudié l'efficacité de 2 types de pessaires : le pessaire anneau avec support et le pessaire Gellhorn et évalué si le type et la taille à utiliser pouvaient être prédits par l'examen clinique. Le pessaire Gellhorn permettait d'obtenir de meilleurs résultats en cas d'antécédents d'hystérectomie et chez les patientes présentant un introït vaginal plus large ( $p=0,005$ et $p=0,01$, respectivement). La taille du pessaire utilisé était également influencée par la largeur du hiatus génital pour les 2 types de pessaire ; la longueur vaginale était positivement associé à la taille du pessaire anneau utilisé. Pour le pessaire Gellhorn, le stade du prolapsus de l'étage moyen $(p=0,006)$ et le stade du prolapsus de l'étage postérieur $(p=0,003)$ étaient également significativement associé à la taille du pessaire utilisé (28).

Plus généralement la tolérance ou encore la satisfaction des patientes pourraient poser question. Récemment une étude de cohorte française, menée auprès de 88 patientes présentant un POP symptomatique visait à évaluer leur satisfaction à 1 mois puis à 6 mois de la pose d'un pessaire anneau. Un taux de satisfaction de l'ordre de $78 \%$ à 1 mois et $84 \%$ à 6 mois étaient retrouvés. Le confort et l'efficacité du pessaire était les principaux avantages rapportés pour près de $60 \%$ des patientes. Dans cette étude également, les auteurs ont cherché à évaluer 
la qualité de vie des patientes en utilisant des questionnaires de symptômes validés. Il existait une amélioration significative de l'ensemble des scores de symptômes urinaires, digestifs et périnéaux (PFDI-20 p < 0,0001, ICIQ-SF p<0,03). (29) Une étude prospective avec un suivi à long terme serait intéressante.

Les perspectives d'avenir sont l'émergence de pessaires nouvelle génération, fabriqués sur mesure avec une imprimante 3D, cependant leur utilisation semble difficile en pratique courante pour le moment (30).

L'association d'un traitement oestrogénique pour les patientes ménopausées était une question assez floue pour les internes. Si l'on en croit la littérature, il semble exister un véritable bénéfice à son utilisation, celle-ci permettant une amélioration de la tolérance et une diminution des complications. Dessie et al. ont ainsi démontré que les femmes qui utilisaient des œstrogènes par voie vaginale avaient une durée de suivi médian plus longue (29,5 mois vs 15,4 mois). Elles étaient moins susceptibles d'arrêter l'utilisation de leur pessaire (30,6\% vs $58,5 \%, \mathrm{P}<0,001)$ et $\mathrm{d}$ 'avoir des pertes vaginales ( $\mathrm{HR}=0,31[0,17-0,58])$. Cependant l'utilisation d'une œstrogénothérapie locale n'était pas protectrice contre les érosions (HR = $0,93[0,54-1,6])$ ou les saignements vaginaux $(\mathrm{HR}=0,78[0,36-1,7])(31)$.

Concernant la surveillance et le rythme de suivi après pose d'un pessaire il n'y a actuellement aucun consensus. Un suivi adapté à la patiente semblait être l'option la plus pertinente pour les internes. Selon la littérature, un suivi tous les 3 à 6 mois est le plus souvent rapporté $(10,11,32,33)$. Clemons et al. proposaient un premier contrôle à 1 semaine puis un suivi tous les 6 à 12 mois si les femmes étaient autonomes ou tous les 2-3 mois dans le cas contraire. Ce mode de suivi nous paraît également favorable (34).

La majorité des internes interrogés semblent ignorer les complications liées au pessaire. Les taux décrits dans la littératures sont très variables allant de entre $1,1 \%$ à $73 \%$, probablement du fait de la disparité de la définition d'une complication (19,35). Les principales 
complications retrouvées sont les saignements, les pertes vaginales, les érosions vaginales, les infections, les douleurs et l'incarcération du pessaire dans la muqueuse vaginale.

Concernant les facteurs d'échecs ou de réussite les avis divergent dans la littérature. Les différents facteurs prédictifs d'échec décrits sont un stade sévère du POP (5), un prolapsus de l'étage postérieur (17), un antécédent d'hystérectomie(20-22) ou de chirurgie génito-urinaire (8), la parité élevé (26), l'IMC bas (20), la béance vulvaire avec un hiatus génital ( $\mathrm{gh})>=5 \mathrm{~cm}$ (36), une longueur vaginale (tvl) <=6 cm (34), une faible activité sexuelle (25), un rapport hiatus génital / longueur totale du vagin supérieur à 0,8 (37) et l'âge plus jeune (29). Pour les facteurs associés à la réussite, le prolapsus de l'étage antérieur avec un OR= 5 (18), l'âge élevé et l'activité sexuelle diminuée étaient le plus fréquemment retrouvés (34).

\section{CONCLUSION}

Bien que les internes semblent globalement sensibilisés à l'utilisation du pessaire, leur connaissance concernant les indications, les complications ou encore la surveillance nous laisse penser qu'il est essentiel de promouvoir leur formation afin que celui-ci fasse partie intégrante de l'arsenal thérapeutique de première intention pour les patientes présentant un POP. La simulation pourrait être un complément de formation pratique intéressant.

L'auteur déclarent ne pas avoir de liens d'intérêts.

\section{BIBLIOGRAPHIE}

1. Bump RC, Mattiasson A, Bø K, Brubaker LP, DeLancey JO, Klarskov P, et al. The standardization of terminology of female pelvic organ prolapse and pelvic floor dysfunction. Am J Obstet Gynecol. juill 1996;175(1):10- 7.

2. Jelovsek JE, Barber MD. Women seeking treatment for advanced pelvic organ prolapse have decreased body image and quality of life. Am J Obstet Gynecol. mai 2006;194(5):1455- 61. 
3. Lowenstein L, Gamble T, Sanses TVD, van Raalte H, Carberry C, Jakus S, et al. Sexual function is related to body image perception in women with pelvic organ prolapse. $\mathbf{J}$ Sex Med. août 2009;6(8):2286- 91.

4. Le Normand L, Cosson M, Cour F, Deffieux X, Donon L, Ferry P, et al. Recommandations pour la pratique clinique : Synthèse des recommandations pour le traitement chirurgical du prolapsus génital non récidivé de la femme par l'AFU, le CNGOF, la SIFUD-PP, la SNFCP et la SCGP. Progrès en Urologie. 1 juill 2016;26:S1 - 7.

5. Wu JM, Matthews CA, Conover MM, Pate V, Jonsson Funk M. Lifetime risk of stress urinary incontinence or pelvic organ prolapse surgery. Obstet Gynecol. juin 2014;123(6):1201- 6.

6. Fritel X, Campagne- Loiseau S, Cosson M, Ferry P, Saussine C, Lucot J-P, et al. Complications after pelvic floor repair surgery (with and without mesh): short-term incidence after 1873 inclusions in the French VIGI-MESH registry. BJOG: An International Journal of Obstetrics \& Gynaecology. 2020;127(1):88- 97.

7. Conquy S, Costa P, Haab F, Delmas V. [Non surgical treatment of prolapse]. Prog Urol. déc 2009;19(13):984- 7.

8. Hanson L-AM, Schulz JA, Flood CG, Cooley B, Tam F. Vaginal pessaries in managing women with pelvic organ prolapse and urinary incontinence: patient characteristics and factors contributing to success. Int Urogynecol J Pelvic Floor Dysfunct. févr 2006; 17(2):155- 9 .

9. Cundiff GW, Weidner AC, Visco AG, Bump RC, Addison WA. A survey of pessary use by members of the American urogynecologic society. Obstet Gynecol. juin 2000;95(6 Pt 1):931- 5 .

10. Pott-Grinstein E, Newcomer JR. Gynecologists' patterns of prescribing pessaries. J Reprod Med. mars 2001;46(3):205- 8.

11. Gorti M, Hudelist G, Simons A. Evaluation of vaginal pessary management: a UKbased survey. J Obstet Gynaecol. févr 2009;29(2):129- 31.

12. Bugge C, Hagen S, Thakar R. Vaginal pessaries for pelvic organ prolapse and urinary incontinence: a multiprofessional survey of practice. Int Urogynecol J. juin

2013;24(6):1017- 24.

13. Velzel J, Roovers JP, Van der Vaart CH, Broekman B, Vollebregt A, Hakvoort R. A nationwide survey concerning practices in pessary use for pelvic organ prolapse in The Netherlands: identifying needs for further research. Int Urogynecol J. oct 2015;26(10):1453- 8.

14. Coelho SA, Brito LGO, Araújo CC de, Aguiar LB, Haddad JM, Giraldo PC, et al. Factors associated with the prescription of vaginal pessaries for pelvic organ prolapse. Clinics (Sao Paulo). 2019;74:e934.

15. Vardon D, Martin-Lasnel M, Agostini A, Fauvet R, Pizzoferrato A-C. Vaginal pessary for pelvic organ prolapse: a survey among french gynecological surgeons. J Gynecol Obstet Hum Reprod. 22 juin 2020;101833.

16. Radnia N, Hajhashemi M, Eftekhar T, Deldar M, Mohajeri T, Sohbati S, et al. Patient Satisfaction and Symptoms Improvement in Women Using a Vginal Pessary for The Treatment of Pelvic Organ Prolapse. J Med Life. sept 2019;12(3):271- 5.

17. Yang J, Han J, Zhu F, Wang Y. Ring and Gellhorn pessaries used in patients with pelvic organ prolapse: a retrospective study of 8 years. Arch Gynecol Obstet. sept 2018;298(3):623- 9.

18. Deng M, Ding J, Ai F, Zhu L. Clinical use of ring with support pessary for advanced pelvic organ prolapse and predictors of its short-term successful use. Menopause. août 2017;24(8):954- 8.

19. Lone F, Thakar R, Sultan AH, Karamalis G. A 5-year prospective study of vaginal 
pessary use for pelvic organ prolapse. Int J Gynaecol Obstet. juill 2011;114(1):56- 9. 20. Yimphong T, Temtanakitpaisan T, Buppasiri P, Chongsomchai C, Kanchaiyaphum S. Discontinuation rate and adverse events after 1 year of vaginal pessary use in women with pelvic organ prolapse. Int Urogynecol J. 2018;29(8):1123- 8.

21. Markle D, Skoczylas L, Goldsmith C, Noblett K. Patient characteristics associated with a successful pessary fitting. Female Pelvic Med Reconstr Surg. sept 2011;17(5):249- 52. 22. Mutone MF, Terry C, Hale DS, Benson JT. Factors which influence the short-term success of pessary management of pelvic organ prolapse. Am J Obstet Gynecol. juill 2005;193(1):89- 94.

23. Komesu YM, Rogers RG, Rode MA, Craig EC, Gallegos KA, Montoya AR, et al. Pelvic floor symptom changes in pessary users. Am J Obstet Gynecol. déc 2007;197(6):620.e1-6.

24. Abdool Z, Thakar R, Sultan AH, Oliver RS. Prospective evaluation of outcome of vaginal pessaries versus surgery in women with symptomatic pelvic organ prolapse. Int Urogynecol J. mars 2011;22(3):273- 8.

25. Brincat C, Kenton K, Pat Fitzgerald M, Brubaker L. Sexual activity predicts continued pessary use. Am J Obstet Gynecol. juill 2004;191(1):198- 200.

26. Fernando RJ, Thakar R, Sultan AH, Shah SM, Jones PW. Effect of vaginal pessaries on symptoms associated with pelvic organ prolapse. Obstet Gynecol. juill 2006;108(1):93- 9. 27. Kuhn A, Bapst D, Stadlmayr W, Vits K, Mueller MD. Sexual and organ function in patients with symptomatic prolapse: are pessaries helpful? Fertil Steril. mai 2009;91(5):1914- 8.

28. Ding J, Song X-C, Deng M, Zhu L. Which factors should be considered in choosing pessary type and size for pelvic organ prolapse patients in a fitting trial? Int Urogynecol J. déc 2016;27(12):1867- 71.

29. Martin Lasnel M, Mourgues J, Fauvet R, Renouf S, Villot A, Pizzoferrato AC. [Patient satisfaction and symptom changes in women using a pessary for pelvic organ prolapse]. Prog Urol. juin 2020;30(7):381- 9.

30. Barsky M, Kelley R, Bhora FY, Hardart A. Customized Pessary Fabrication Using Three-Dimensional Printing Technology. Obstet Gynecol. 2018;131(3):493- 7.

31. Dessie SG, Armstrong K, Modest AM, Hacker MR, Hota LS. Effect of vaginal estrogen on pessary use. Int Urogynecol J. sept 2016;27(9):1423 - 9.

32. O'Dell K, Atnip S, Hooper G, Leung K. Pessary Practices of Nurse-Providers in the United States. Female Pelvic Med Reconstr Surg. août 2016;22(4):261 - 6.

33. Khaja A, Freeman RM. How often should shelf/Gellhorn pessaries be changed? A survey of IUGA urogynaecologists. Int Urogynecol J. juill 2014;25(7):941 - 6.

34. Clemons JL, Aguilar VC, Tillinghast TA, Jackson ND, Myers DL. Patient satisfaction and changes in prolapse and urinary symptoms in women who were fitted successfully with a pessary for pelvic organ prolapse. Am J Obstet Gynecol. avr 2004;190(4):1025 - 9.

35. Bai SW, Yoon BS, Kwon JY, Shin JS, Kim SK, Park KH. Survey of the characteristics and satisfaction degree of the patients using a pessary. Int Urogynecol J Pelvic Floor Dysfunct. juin 2005;16(3):182- 6; discussion 186.

36. Mao M, Xu T, Kang J, Zhang Y, Ai F, Zhou Y, et al. Factors associated with longterm pessary use in women with symptomatic pelvic organ prolapse. Climacteric. oct 2019;22(5):478- 82.

37. Geoffrion R, Zhang T, Lee T, Cundiff GW. Clinical characteristics associated with unsuccessful pessary fitting outcomes. Female Pelvic Med Reconstr Surg. déc 2013;19(6):339- 45. 
$\stackrel{13}{\text { Page } 13 \text { of } 13}$ 